\title{
Genome-wide association study of appendicular lean mass in UK Biobank cohort
}

2 Yu-Fang Pei ${ }^{\# 1,2}$, Yao-Zhong Liü ${ }^{\# 3}$, Xiao-Lin Yang ${ }^{\# 2,4}$, Hong Zhang ${ }^{2,4}$, Gui-Juan Feng ${ }^{1,2}$, Lei Zhang ${ }^{2,4}$

$3{ }^{1}$ Department of Epidemiology and Health Statistics,

$4 \quad{ }^{2}$ Jiangsu Key Laboratory of Preventive and Translational Medicine for Geriatric Diseases, School of Public Health,

5 Medical College of Soochow University, Jiangsu, PR China.

$6{ }^{3}$ Department of Biostatistics and Data Science, Tulane University School of Public Health and Tropical Medicine,

7 New Orleans, LA, USA.

$8{ }^{4}$ Center for Genetic Epidemiology and Genomics, School of Public Health, Medical College of Soochow University,

9 Jiangsu, PR China.

10 Keywords: appendicular lean mass, GWAS, UK Biobank

11 Running title: GWAS of appendicular lean mass

12 \#: These authors contributed equally to this work.

13 Corresponding author:

14 Lei Zhang, Ph.D.

15 Associate Professor

16 Center for Genetic Epidemiology and Genomics

17 School of Public Health, Medical College, Soochow University

18199 Ren-ai Rd., SuZhou City, Jiangsu Province 215123, PR China

19 Tel: (86)0512-65883871 Email: lzhang6@suda.edu.cn 


\section{Abstract}

22 Lean body mass (LBM), an important physiological measure, has a strong genetic determination.

23 To clarify its genetic basis, a large-scale genome-wide association study (GWAS) of

24 appendicular lean mass (ALM) was conducted in 450,580 UK Biobank subjects. A total of 717

25 variants $\left(\mathrm{p}<5 \times 10^{-9}\right)$ from 561 loci were identified, which were replicated across genders

26 (achieving $\mathrm{p}<5 \times 10^{-5}$ in both genders). The identified variants explained $\sim 11 \%$ phenotypic

27 variance, accounting for one quarter of the total $40 \%$ GWAS-attributable heritability. The

28 identified variants were enriched in gene sets related to musculoskeletal and connective tissue

29 development. Of interest are several genes, including ADAMTS3, PAM, SMAD3 and MEF2C,

30 that either contain multiple significant variants or serve as the hub genes of the associated gene

31 sets. Polygenic score prediction based on the associated variants was able to distinguish subjects

32 of high from low ALM. Overall, our results offered significant findings on the genetic basis of

33 lean mass through an extraordinarily large sample GWAS. The findings are important to not only

34 lean mass per se but also other complex diseases, such as type 2 diabetes and fracture, as our

35 Mendelian randomization analysis showed that ALM is a protective factor for these two diseases. 


\section{Introduction}

Lean body mass (LBM) is an important physiological index. The decline of LBM with aging,

also known as sarcopenia, is a critical factor for functional impairment and physical disability and a major modifiable cause of frailty in the elderly $[1,2]$. LBM is associated with bone mineral

41 density (BMD), and hence may be also relevant to risk for osteoporosis [3]. Other LBM-related

42 conditions include dysmobility syndrome [4], sarcopenic obesity [5], and cachexia [6]. Overall,

43 sarcopenia was responsible for an increased risk of mortality, with a hazard ratio of 1.29 to 2.39

44 [7].

45 LBM has a significant genetic component, as evidenced by a high heritability of $50 \%$ to $80 \%$

46 as observed in twin studies [8, 9]. However, findings on specific genes for human lean mass

47 variation remain limited even with the powerful genome-wide association study (GWAS)

48 approach. A key reason for the limited findings, as in other human complex traits, is the modest

49 sample size used in most GWAS so far performed in LBM [10-14], resulting in few SNPs (single

50 nucleotide polymorphisms) identified with genome-wide significance.

51 As a notable example, a recent large meta-analysis of GWAS amassed 20 cohorts of European

52 ancestry with a total sample size of $>38,000$ for whole body lean mass (WBM) and of $>28,000$

53 for appendicular lean mass (ALM) [15]. However, despite of the large sample used, the percent

54 variance explained by the identified SNPs was still only $0.23 \%$ and $0.16 \%$ for WBM and ALM,

55 respectively, suggesting that most of the heritability of LBM was still undetected. Therefore,

56 even with such a large GWAS meta-analysis, it is still necessary to boost the sample size further

57 so as to enhance the statistical power for detecting more causal SNPs underlying LBM.

58 Here in this study, with a sample containing half-million subjects of European origin from

59 UK Biobank (UKB), we performed a GWAS of appendicular lean mass (ALM). At the stringent 
60 genome-wide significance level $\left(\mathrm{p}<5 \times 10^{-9}\right)$, we identified $>700$ variants that were replicated

61 across genders. Our findings revealed a large number of genetic variants for LBM and

62 contributed to the characterization of the genetic architecture of this important complex trait.

63 Through this GWAS we demonstrated the power for mapping the genetic landscape of common

64 human complex traits/diseases using extraordinarily large samples. 
Results

Basic characteristics of the studied UKB sample are listed in Supplementary Table 1. In this study, we quantified appendicular lean mass (ALM) by appendicular fat-free mass measured by

69 electronic impedance. This measurement of lean mass is reliable based on its strong correlation

70 with ALM measured by DXA in 4,294 UKB subjects (with a Pearson's correlation coefficient of

$\left.710.96, \mathrm{p}<2.2 \times 10^{-16}\right)$.

\section{Main association results}

73 Raw ALM was adjusted with appendicular fat mass (AFM) and the adjusted ALM (ALM adj $)$

74 was the phenotype used for the GWAS. Following quality control (QC) of both ALM $_{\text {adj }}$ and

75 genome-wide genotypes, data from 19.4 million variants with minor allele frequency

76 (MAF) $>0.1 \%$ and imputation quality score $>0.3$ were available in 244,945 female and 205,635

77 male subjects.

78 In each gender group, additive effect of each variant was tested on ALM adj with BOLT-LMM

79 [16], controlling for age, age ${ }^{2}$, height and height ${ }^{2}$. The genomic inflation factor showed notable

80 inflation in both gender groups $\left(\lambda_{\text {female }}=1.92, \lambda_{\text {male }}=1.77\right)$. To examine observed inflation for

81 potential polygenic effects and other biases, linkage disequilibrium score regression (LDSC)

82 analysis was performed [17]. The estimated mean chi-square and intercept were 2.34 and 1.12

83 for females, and 2.53 and 1.15 for males, corresponding to an attenuation ratio (AR) of 0.098 and

840.090 , respectively. The AR estimates are comparable to those estimated in the subset of 369,968

85 unrelated British white subjects (0.090 and 0.074 for females and males, respectively), who were

86 extracted from the total sample.

87 Using BOLT-REML [18], GWAS-attributable heritability was estimated, which was 0.381

88 (s.e $3.30 \times 10^{-3}$ ) and $0.394\left(\right.$ s.e $3.80 \times 10^{-3}$ ) in females and males, respectively. LDSC estimated a 
89 genetic correlation coefficient as high as 0.90 (s.e 0.01 ) between the two genders, implying that

90 most GWAS-attributable heritability was shared across genders.

91 Given the shared heritability across genders, across-gender meta-analysis was performed with

92 the inverse variance weighted fixed-effects model to combine the gender-specific GWAS results.

93 The meta-analysis signals have an $\mathrm{AR}$ of 0.115 (mean chi-square=3.69, intercept=1.31).

94 Genome-wide significance (GWS) level was set to $\alpha=5 \times 10^{-9}$, and a suggestive significance level

95 was set to $\alpha=5 \times 10^{-5}$. An association was declared to be "replicated" if it is 1) significant at the

96 GWS level in the across-gender meta-analysis and 2) significant at the suggestive level within

97 each gender.

98 Based on the above criteria, a total of 589 loci were identified at GWS level in across-gender 99 meta-analysis $\left(\mathrm{p}<5 \times 10^{-9}\right)$, which were also replicated $\left(\mathrm{p}<5 \times 10^{-5}\right)$ across genders. To check 100 potential linkage disequilibrium (LD) among these loci, LD analysis was performed on 589 lead

101 variants (each from one of the loci). It was found that 47 lead variants are not in linkage 102 equilibrium with each other $\left(\mathrm{LD} \mathrm{r}^{2}>0.1\right)$ due to long-range LD. After removing 28 loci, the lead 103 variants in the remaining 561 loci were all in linkage equilibrium (LD $\left.r^{2}<0.1\right)$. Therefore, these 104561 loci were treated as independent loci for downstream analysis.

105 Approximate conditional association analysis and across-gender meta-analysis were 106 recursively performed, which further identified an additional set of 156 conditionally significant 107 variants $\left(\mathrm{p}<5 \times 10^{-9}\right.$ in across gender meta-analysis) that were replicated across genders $\left(\mathrm{p}<5 \times 10^{-5}\right.$ 108 within each gender). These additional variants were also in linkage equilibrium (LD $\left.r^{2}<0.1\right)$ with 109 the lead variants of the 561 loci.

110 In total, 717 (i.e., 561+156) independent variants from 561 distinct loci were associated with $111 \mathrm{ALM}_{\mathrm{adj}}$ (Supplementary Table 2). Among the 717 lead variants, 172 achieved the strongest 
112 significance level $\left(\mathrm{p}<5 \times 10^{-9}\right)$ in both genders (categorized here as the Tier 1 variants). Also, 144

113 variants achieved $\mathrm{p}$ values $<5 \times 10^{-9}$ in females, and $\mathrm{p}$ values $<5 \times 10^{-5}$ in males; 62 variants

114 achieved $\mathrm{p}$ values $<5 \times 10^{-9}$ in males, and $\mathrm{p}$ values $<5 \times 10^{-5}$ in females (categorized here as the

115 Tier 2 variants). At last, 339 variants achieved $\mathrm{p}$ values $<5 \times 10^{-5}$ in both genders and $\mathrm{p}$ values $<$

$1165 \times 10^{-9}$ in across-gender meta-analysis (categorized here as the Tier 3 variants).

117 Of the above identified loci, 17 were reported by GWAS or meta-analysis of DXA-derived 118 lean mass [13, 15, 19]; 104 were reported by a study of electronic impedance measured lean 119 mass in a subset UKB cohort subjects $(\mathrm{N}=155,961)[20]$.

120 We also evaluated the overlap of the identified loci with those identified for several obesity

121 traits, including body mass index (BMI), waist circumference (WC), WC adjusted for BMI

122 (WC $\left.\mathrm{Wdj}_{\mathrm{ad}} \mathrm{BMI}\right)$, waist-hip ratio (WHR) and WHR adjusted for BMI (WHR adjBMI). SNPs in 302

123 loci (defined as the lead SNP + $500 \mathrm{~kb}$ flanking at each side) showed association with one or

124 more obesity traits at the conventional significance level $5.0 \times 10^{-8}$, while no trait was associated 125 with the remaining 259 loci, demonstrating their novelty and possibly, specificity to lean, but not 126 fat mass.

\section{Gender heterogeneity/specificity}

128 In addition to the 561 loci that are replicated across genders, our analysis also identified 152

129 loci that are significant $\left(\mathrm{p}<5 \times 10^{-9}\right)$ in across-gender meta-analysis but not significant at the 130 suggestive level $\left(\mathrm{p}<5 \times 10^{-5}\right)$ in either gender group (Supplementary Table 3). These loci may 131 represent gender specific signals pending further replication.

132 Of the 717 identified variants (of the 561 loci), $109(15.2 \%)$ have a high level across-gender 133 meta-analysis heterogeneity $\left(\mathrm{I}^{2}>50 \%\right.$ ), all (except one) of which belong to Tiers 1 or 2 variants. 134 A statistical test on gender difference in allele effect size showed that the difference is significant 
135 in only 2 SNPs, $r s 2972156\left(\mathrm{p}_{\text {diff }}=2.49 \times 10^{-12}\right)$ and $\mathrm{rs} 1933801\left(\mathrm{p}_{\text {diff }}=4.65 \times 10^{-6}\right)$, after accounting

136 for multiple testing $\left(\alpha=0.05 / 717=6.97 \times 10^{-5}\right)$, suggesting that almost all of the identified variants

137 may have similar effect sizes across genders. The two SNPs (rs2972156 and rs1933801) with

138 different effect size between genders achieved $\mathrm{p}$ values of $1.30 \times 10^{-46}$ and $2.40 \times 10^{-26}$,

139 respectively, in males and p values of $4.20 \times 10^{-7}$ and $1.30 \times 10^{-6}$, respectively, in females.

140 Heritability distribution

141 The 717 identified variants include 654 common variants (MAF>5\%), 52 less common

142 variants $(5 \% \geq \mathrm{MAF}>1 \%)$ and 11 rare variants $(\mathrm{MAF} \leq 1 \%)$. Collectively, these variants explain

$14310.82 \%$ phenotypic variance in the total sample, most of which $(9.91 \%)$ is accounted for by

144 common variants. As expected, variants with a smaller MAF generally have a larger per allele

145 effect size (Figure 1). For example, the average per allele effect size in rare variants (mean 0.11,

146 s.d 0.05) is 6-fold larger than common variants (mean 0.02, s.d 0.007).

147 Applying the stratified LDSC analysis, the explained heritability was partitioned into 24

148 functional categories [21]. Statistically significant enrichments were observed for 19 functional

149 categories ( $\mathrm{p}<0.05 / 24$, Figure 2). In line with the observations by Finucane et al. [21], regions

150 conserved in mammals showed the strongest enrichment of any category, with $2.6 \%$ of SNPs

151 explaining an estimated $34.5 \%$ of SNP heritability (enrichment ratio $\left.(\mathrm{EA})=13.2, \mathrm{P}=3.39 \times 10^{-19}\right)$.

152 Other categories with significant enrichment included coding regions $\left(\mathrm{EA}=8.8, \mathrm{P}=1.76 \times 10^{-7}\right), 3^{\prime}$

153 UTR $\left(\mathrm{EA}=5.7, \mathrm{P}=3.73 \times 10^{-4}\right)$, transcription starting site $\left(\mathrm{EA}=5.1, \mathrm{P}=1.71 \times 10^{-5}\right)$, and $\mathrm{H} 3 \mathrm{~K} 9 \mathrm{ac}$

154 histone marks $\left(\mathrm{EA}=5.1, \mathrm{P}=2.07 \times 10^{-15}\right)$. Neither promoter nor 5'-UTR region showed significant 155 enrichment, though 5'-UTR region had a high estimate of EA (15.5, p=0.03).

156 A new function of the stratified LDSC method was used to assess focal tissues for heritability 157 enrichment [22], using two gene expression datasets [23, 24]. A total of 19 tissues/cells are 
enriched at a false discovery rate (FDR) $<5 \%$ (Figure 2). About half (9) of them belong to

159 musculoskeletal and connective system, including cartilage, chondrocytes, osteoblasts,

160 fibroblasts, smooth muscle, myometrium, cervical vertebrae, synovial membrane and stromal

161 cells.

\section{Candidate genes prioritization}

163 To prioritize candidate genes at the associated loci, we used multiple analytical strategies. A 164 set of credible risk variants (CRVs) at each locus were defined as variants with high LD with the 165 lead variant $\left(r^{2}>0.8\right)$. A total of 17,968 CRVs were defined (Supplementary Table 4). Based on 166 the CRVs, 6 types of supporting evidence were used to prioritize 1,337 candidate genes.

167 (Supplementary Tables 5-10).

168 A number of genes have multiple lines of supporting evidence. Peptidylglycine 169 Alpha-Amidating Monooxygenase $(P A M)$ at 5q21.1, in particular, has all lines of supporting 170 evidence. This locus contains two independent signals. The first is a mis-sense rare SNP 171 rs78408340 $\left(\mathrm{MAF}=0.01 \%, \mathrm{p}=6.10 \times 10^{-10}\right)$ inside PAM, and the second is a common SNP 172 rs400596 located between PAM (129.5 kb from PAM) and SLCO6Al genes (237.2 kb from

173 SLC06A1). Polymorphisms at rs400596 are associated with the PAM expression level in whole 174 blood $\left(p=2.51 \times 10^{-21}\right)$ [23] and associated with its protein level in peripheral blood ( $p=$ $\left.175 \mathrm{p}=2.51 \times 10^{-30}\right)[25] . P A M$ is also prioritized by both SMR [26] and DEPICT [27], strengthening 176 its functional relevance.

\section{Comparison between imputation and sequencing-based association signal}

178 Of the 717 identified variants, 42 are mis-sense coding ones. Forty of them, including 7 rare

179 ones, are available in the recently released UKB exome sequencing data that contain a subset of $180 \sim 50,000$ subjects from the whole UKB cohort. Using a set of 45,554 unrelated European subjects 
181 who were both genotyped/imputed and sequenced, we compared the imputation-based

182 association results with exome sequencing based results. The 7 rare variants appeared to have

183 limited imputed dosage variation hence their imputation association p-values were not able to

184 derive. In the sequencing data, 3 of these 7 variants were nominally significant $(\mathrm{p}<0.05$,

185 Supplementary Table 11), suggesting limited power in imputation-based association analysis

186 (compared with sequence-based analysis) for rare variants. This limited power may be alleviated

187 by increased sample size since in the whole UKB cohort these 7 rare variants achieved

188 significant $\mathrm{p}$ values in imputation-based association analysis.

189 Of the remaining 33 variants, the imputation-based and sequencing-based p-values were

190 highly concordant. For example, the imputation-based p-values are within 2-fold difference of

191 the sequencing-based p-values for up to 29 variants. Overall, these observations support that

192 imputation-based association signals are close to the real sequencing-based association signals in

193 a large sample. Therefore, imputation based GWAS may be able to identify true associations,

194 even for those of rare variants.

195 Mis-sense variants and the associated genes

196 As mentioned above, of the 717 identified variants, 42 are mis-sense coding ones. Majority of

197 these 42 mis-sense mutations are predicted to be deleterious according to more than one

198 bioinformatics tool including PolyPhen2 [28], SIFT [29], PROVEAN [30] and Fathmm [31]

199 (Supplementary Table 12), supporting their functional relevance.

200 Mis-sense mutations are enriched among rare variants. Eight of the 11 rare variants are

201 mis-sense mutations, which is in clear contrast to 34 mis-sense mutations among the remaining

202706 variants (odds ratio $(\mathrm{OR})=55.37$, Fisher's exact test $\mathrm{p}=7.11 \times 10^{-9}$ ). Evidence of the

203 enrichment became stronger by comparing 21 mis-sense mutations from 63 rare or less common 
variants vs. 22 mis-sense mutations from 654 common variants $(\mathrm{OR}=14.36$, Fisher's exact test

$\left.205 \mathrm{p}=5.75 \times 10^{-13}\right)$, suggesting that low frequency mutations are more likely to play a direct role in

206 changing protein function.

207 Genes containing mis-sense variants are listed in Table 1. In particular, the ADAMTS3 gene

208 contains 3 rare or less common mis-sense variants (rs141374503 MAF=0.4\% $\mathrm{p}=2.02 \times 10^{-27}$;

$209 \mathrm{rs} 150270324 \mathrm{MAF}=1.3 \%, \mathrm{p}=2.36 \times 10^{-14}$, and $\mathrm{rs} 139921635 \mathrm{MAF}=2.4 \%, \mathrm{p}=4.06 \times 10^{-15}$ ). In

210 addition, it also contains multiple non-mis-sense variants, including 3 conditionally significant

211 variants in its intron region: $r 572653979\left(\mathrm{MAF}=7.8 \%, \mathrm{p}=9.51 \times 10^{-11}\right), \mathrm{rs} 78862524(\mathrm{MAF}=5.5 \%$,

$\left.212 \mathrm{p}=3.24 \times 10^{-23}\right)$ and $\mathrm{rs} 769821342\left(\mathrm{MAF}=3.2 \%, \mathrm{p}=1.27 \times 10^{-14}\right)$, and 2 in its flanking inter-genic

213 region: $\mathrm{chr} 4: 73496010\left(\mathrm{MAF}=47 \%, \mathrm{p}=3.87 \times 10^{-21}\right)$ and $\mathrm{rs} 10518106\left(\mathrm{MAF}=6 \%, \mathrm{p}=1.16 \times 10^{-83}\right)$.

214 Though these SNPs are $367.0 \mathrm{~kb}$ apart at most, they are in linkage equilibrium with each other

215 (LD $\left.\mathrm{r}^{2}<0.1\right)$. Together, the 8 variants from the ADAMTS3 gene explain $0.18 \%$ of phenotypic

216 variance, making this region the most contributive locus.

\section{Gene-based and gene set enrichment analyses}

218 A total of 3,101 genes were significant at the gene-based genome-wide significance level $219\left(\alpha=0.05 / 19,098=2.62 \times 10^{-6}\right.$, Supplementary Table 13 $)$, and 85 gene sets were significant at the 220 gene set significance level $\left(\alpha=0.05 / 10,655=4.69 \times 10^{-6}\right.$, Supplementary Table 14).

221 The most significant gene set is GO:0001501 'skeletal system development' $\left(\mathrm{p}=8.88 \times 10^{-24}\right)$,

222 followed by GO:0031012 'proteinaceous extracellular matrix' $\left(\mathrm{p}=5.01 \times 10^{-14}\right)$, GO:0061448

223 'connective tissue development' $\left(\mathrm{p}=1.10 \times 10^{-13}\right)$, GO:0048705 'skeletal system morphogenesis'

$224\left(\mathrm{p}=9.33 \times 10^{-13}\right)$ and GO:0031012 'extracellular matrix' $\left(\mathrm{p}=1.05 \times 10^{-12}\right)$. Additional gene sets with

225 known function related to musculoskeletal and connective system, such as GO:0051216: 
226 'cartilage development' $\left(\mathrm{p}=3.30 \times 10^{-12}\right)$ and GO:0042692 'muscle cell differentiation'

$227\left(\mathrm{p}=1.45 \times 10^{-6}\right)$, were also identified.

228 Genes involved in multiple gene sets are likely to act as hub genes and may play a central

229 regulatory role. From the list of significant gene sets, the most frequently involved gene is

230 SMAD3 (gene-based association $\mathrm{p}=8.11 \times 10^{-42}$ ), which was involved in 46 out of the 85

231 significant gene sets. It was followed by SOX9 ( $\mathrm{p}=0.05$, in 44 gene sets), MEF $2 C\left(\mathrm{p}=2.83 \times 10^{-9}\right.$,

232 in 42 gene sets) and BMP4 ( $\mathrm{p}=0.15$, in 42 genes). All these 4 genes were reported by previous

233 studies as important candidate genes for muscle development [32-35]. However, SOX9 is only

234 nominally significant and BMP4 is not significant at single gene level, indicating that the

235 significant pathway signals may not be contributed by the two genes. Altogether, there are 34

236 genes, each of which was involved in more than 30 of the 85 significant gene sets.

237 Protein-protein interaction (PPI) analysis using these 34 hub genes connects them into a tight

238 interactional network (Figure 3). This network contains multiple genes that are important for

239 skeletal muscle development, such as TGF signaling pathway genes (TGFB1, TGFB2 and

$240 T G F B R 2)$, BMP signaling pathway genes (BMP2 and BMP4) and SMAD family genes (SMAD1,

241 SMAD2, SMAD3 and SMAD4).

$242 \quad$ Polygenic risk score profiling

243 To assess the ability of the GWAS findings to predict ALM, a polygenic scoring analysis was

244 performed in the subset of 369,968 unrelated British white subjects from the UKB cohort. Three

245 quarters of the subjects (277,762 participants, including 149,329 females) were randomly

246 selected as the training sample, with the remaining subjects (92,206 participants, including

24749,660 females) as the validation sample. 
The training sample identifies 72,456 variants that achieved a $p$-value $<1 \times 10^{-5}$ for association with $\mathrm{ALM}_{\mathrm{adj}}$. Using these variants as predictor, the predicted genome-wide polygenic score (GPS) and the real phenotype residual in the validation sample are significantly correlated (Pearson's

251 correlation coefficient $0.22,95 \%$ CI $\left.(0.21,0.22), \mathrm{p}<2.2 \times 10^{-16}\right)$. Mean phenotype residuals in the 252 top tail are significantly higher than that in the bottom tail of the GPS distribution (Figure 4).

253 For example, the predicted top 1\% subjects have an increased average residual of 1.16 than the 254 predicted bottom $1 \%$ participants $(0.57$ (s.d 0.96) vs. -0.59 (s.d 0.94)), corresponding to an 1.69 255 kilo-gram (kg) increase of raw ALM (24.61 kg (s.d $5.89 \mathrm{~kg})$ vs. $22.92 \mathrm{~kg}$ (s.d $5.27 \mathrm{~kg})$ ). In the 256 female group, the predicted top $1 \%$ participants have on average $1.39 \mathrm{~kg}$ increase of raw ALM 257 than the predicted bottom $1 \%$ participants $(20.26 \mathrm{~kg}(\mathrm{~s} . \mathrm{d} 2.75 \mathrm{~kg}) \mathrm{vs} .18 .87 \mathrm{~kg}(\mathrm{~s} . \mathrm{d} 2.45 \mathrm{~kg})) . \mathrm{In}$ 258 males, the increase is $2.29 \mathrm{~kg}(29.82 \mathrm{~kg}$ (s.d $4.18 \mathrm{~kg})$ vs. $27.53 \mathrm{~kg}$ (s.d $3.56 \mathrm{~kg})$ ). These results 259 demonstrate that the GPS prediction based on the current GWAS finding is capable of 260 identifying subjects of high or low levels of ALM.

\section{Genetic correlations with other traits}

262 To test whether lean mass has a shared genetic etiology with other diseases and relevant traits, 263 a genetic correlation analysis was performed with the LDSC method [17]. Here, ALM studied in 264 our study is strongly genetically correlated with DXA-derived whole body lean mass and the 265 ALM, which were studied by a previous GWAS meta-analysis [15] ( $\mathrm{r}_{\mathrm{g}}=0.87$ and 0.78$)$ (Figure 266 5). Furthermore, ALM is modestly correlated with BMI $\left(r_{g}=0.31\right)$. However, the correlation with 267 BMD is low $\left(r_{g}=0.05\right)$. ALM is most negatively correlated with BMI adjusted leptin $\left(r_{\mathrm{g}}=-0.41\right)$. It 268 is also negatively correlated with body fat $\left(r_{g}=-0.17\right)$, suggesting a reverse developmental 269 direction towards lean and fat mass.

270 Mendelian randomization analysis 
271 To investigate whether ALM is causally linked with other complex diseases, a Mendelian

272 randomization analysis was performed with GSMR [26]. Ten diseases from a variety of

273 categories were chose for evaluation, including fracture [36], type 2 diabetes (T2D) [37], asthma

274 [38], insomnia [39], inflammatory bowel disease (IBD) [40], smoking addiction [41], coronary

275 artery disease (CAD) [42], amyotrophic lateral sclerosis (ALS) [43], bipolar disorder [44] and

276 autistic spectrum disorder (ASD) [45]. At the corrected significance level $5 \times 10^{-3}(0.05 / 10)$, ALM

277 is causally associated with type 2 diabetes $\left(\mathrm{T} 2 \mathrm{D}, \mathrm{p}=4.38 \times 10^{-8}\right)$ and fracture $\left(\mathrm{p}=1.18 \times 10^{-3}\right)$, but

278 not with any other disease (Supplementary Table 15). Specifically, a negative association is

279 observed between ALM and both diseases, indicating that ALM is a protective factor for both

280 diseases. For T2D, an increase in the unit of one s.d of ALM residual corresponds to a decreased

281 OR of $0.91(95 \%$ CI [0.88, 0.94]). For fracture, an increase in the unit of one s.d. of ALM

282 residual corresponds to a decreased OR of 0.95 (95\% CI [0.92, 0.98]). 


\section{Discussion}

285 The incapacity in GWAS to detect and replicate specific genetic variants for human complex

286 traits, contradicting to a trait's established high heritability, e.g., height, was formally recognized

287 as the "missing heritability" problem a decade ago [46, 47]. An explanation is the so called

288 "polygenic model", where hundreds or even thousands of common SNP variants act additively,

289 with each contributing only a "tiny" fraction of the trait variation. The effect of each individual

290 variant is so small that a GWAS with a limited sample size $(n<20,000)$ may be extremely

291 difficult, if not impossible, to detect (let alone replicate) a variant at the genome-wide

292 significance threshold.

293 The polygenic model was supported by the genome-wide complex trait analysis (GCTA),

294 where trait similarity among unrelated subjects was correlated with and explained to a large

295 fraction by similarity of common SNPs at genome-wide scale [48]. Furthermore, with sample

296 sizes at the scale of hundreds of thousands, two GWAS indeed identified at genome-wide

297 significance 700 variants for adult height [49] and >100 loci for schizophrenia [50]. The

298 successful stories offer a promising prospect for a GWAS with an extraordinarily large sample

299 size to ultimately unravel the puzzling genetic architecture for human complex traits and

300 common diseases.

301 In this study of lean mass with around half million subjects, the largest sample used for

302 GWAS of lean mass so far, a successful endeavor was accomplished again. More than 700

303 variants were identified at the significance of genome-wide scale $\left(\mathrm{p}<5 \times 10^{-9}\right)$. In particular, more

304 than half of these variants achieved genome-wide significance $\left(\mathrm{p}<5 \times 10^{-9}\right)$ in one gender and

305 were replicated also in another gender $\left(\mathrm{p}<5 \times 10^{-5}\right)$. Overall, these $>700$ variants contributed $\sim 11 \%$ 
of ALM variation, again, the largest explainable fraction of variation for lean mass reported so

307 far in a GWAS.

308 Our findings of $>700$ variants are expected for a complex trait with a high heritability, 309 particularly considering another trait with comparable heritability, height, which detected also $310 \sim 700$ variants [49]. Interestingly, the majority loci in previous smaller GWAS [13] or 311 meta-analysis [15] of lean mass are also significant in the present study, providing replication 312 evidence from independent samples.

313 The functional relevance of our identified variants is supported by the gene set enrichment 314 analysis, where GO terms, including GO:0001501 'skeletal system development', GO:0061448

315 'connective tissue development', GO:0051216 'cartilage development' and GO:0042692 'muscle 316 cell differentiation', are among the top gene sets of significance. Specifically, the "hub genes" 317 involved in these terms are tightly connected into a network that contains TGF pathway genes, 318 BMP pathway genes and SMAD family genes, which are all important musculoskeletal 319 development genes/pathways. This finding is concordant with developmental biology since cells 320 from bone, cartilage, muscle and fat share the same progenitor, the mesenchymal stem cells, and 321 pleiotropy of muscle and bone is well recognized in both humans [51] and animal models [52].

322 Among the variants identified, those of several genes, such as SMAD3, MEF2C, ADAMTS3 323 and $P A M$, are interesting and may need further investigation. The first two genes are the hub 324 genes involved in half of the significant enriched gene sets. The third gene, ADAMTS3, contains 3258 variants, including 3 rare or less common mis-sense mutations, which in total explains $\sim 0.2 \%$ 326 of ALM variation. The fourth gene, PAM, has multiple lines of supporting evidence for its 327 regulatory roles, e.g., containing a mis-sense rare SNP rs78408340 $\left(\mathrm{MAF}=0.01 \%, \mathrm{p}=6.10 \times 10^{-10}\right)$. 328 An intergenic variant, rs400596, is associated with the PAM expression level in whole blood 
$329\left(\mathrm{p}=2.51 \times 10^{-21}\right)[23]$ and associated with its protein level in peripheral blood tissue $(\mathrm{p}=$ $330 \mathrm{p}=2.51 \times 10^{-30}$ ) [25]. These genes may represent the next candidates for functional and

331 mechanistic analysis of lean mass regulation.

332 In summary, we performed a GWAS using half-million subjects for lean mass. Owing to its

333 high statistical power, our study identified a large number of variants mapped to GO terms with

334 functional relevance to musculoskeletal development. The explained variation of $\sim 11 \%$ of lean 335 mass by the identified variants represents a significant leap in revealing the "hidden" heritability 336 of this complex trait using GWAS. Our findings' translational value is marked by lean mass' 337 importance to other complex diseases, such as type 2 diabetes and fracture, as our Mendelian 338 randomization analysis showed that ALM is a protective factor for these two diseases. Overall, 339 our study provides another example, where GWAS of substantially increased sample size may 340 lead a way to ultimately and thoroughly delineate genetic architecture of human complex traits.

341 This epitomizes the value of big data in genetic research of humans. 
Materials and Methods

\section{Study participants}

345 Study sample came from the UK Biobank (UKB) cohort, which is a large prospective cohort

346 study of $\sim 500,000$ participants from across the United Kingdom, aged between 40-69 at

347 recruitment. Ethics approval for the UKB study was obtained from the North West Centre for

348 Research Ethics Committee (11/NW/0382), and informed consent was obtained from all

349 participants. This study (UKB project \#41542) was covered by the general ethical approval for

350 the UKB study.

351 All the included subjects are those who self-reported as white (data field 21000). Subjects

352 who had a self-reported gender inconsistent with the genetic gender, who were genotyped but not

353 imputed or who withdraw their consents were removed. The final sample consisted of 450,580

354 subjects, including 244,945 females and 205,635 males.

\section{$355 \quad$ Phenotype and modeling}

356 Body composition was measured by bioelectrical impedance approach. Appendicular lean

357 mass (ALM) was quantified by the sum of fat-free mass at arms (data fields 23121 and 23125)

358 and at legs (data fields 23113 and 23117). Appendicular fat mass (AFM) was quantified by the

359 sum of fat mass at arms (data fields 23120 and 23124) and at legs (data fields 23112 and 23116).

360 In each gender, covariates including AFM, age, age ${ }^{2}$, height and height ${ }^{2}$ were tested for

361 significance in association with ALM using a step-wise linear regression model implemented in

362 the R function stepAIC. Raw ALM values were adjusted by the significant covariates, and the

363 residuals were normalized into inverse quantiles of standard normal distribution, which were

364 used for subsequent association analysis. 
A small subset of 4,294 subjects also received a dual-energy X-ray absorptiometry (DXA) body composition scan, and hence their DXA-derived ALM is also available. Therefore, raw

367 ALM derived from DXA and from electric impedance was compared in these subjects by

368 Pearson's correlation coefficient.

Genotype quality control

370 Genome-wide genotypes for all subjects were available at 784,256 genotyped autosome 371 markers, and were imputed into UK10K haplotype, 1000 Genomes project phase 3 and 372 Haplotype Reference Consortium (HRC) reference panels. A total of $\sim 92$ million variants were 373 generated by imputation. We excluded variants with $\mathrm{MAF}<0.1 \%$ and with imputation $\mathrm{r}^{2}<0.3$. As 374 a result, $~ 19.4$ million well imputed variants were retained for subsequent genetic association 375 analysis.

\section{Genetic association analysis}

377 In each gender group, we used BOLT-LMM to perform linear mixed model (LMM) analysis 378 [16]. As the LMM analysis can adjust for population structure and relatedness, we included all 379 eligible subjects into analysis, as recommended by BOLT [53]. We did not include principal 380 components (PCs) of ancestry as covariates in the LMM analysis.

381 After sex-specific associations were analyzed, we meta-analyzed the summary statistics of the

382 two genders by inverse-variance weighted fixed-effects model with METAL [54]. The

383 genome-wide significance (GWS) level was set at $\alpha=5 \times 10^{-9}$, to account for both common and

384 rare variants. The variants that passed this threshold in across-gender meta-analysis were then 385 checked for replicability across genders based on a suggestive significance level $5 \times 10^{-5}$ in each 386 gender. The suggestive level was set so as to account for multiple testing of presumed maximal 387 number of 1000 independent loci (0.05/1000). An association was defined as "replicated" if the 
388 signal was significant at the GWS level $\left(\mathrm{p}<5 \times 10^{-9}\right)$ in the meta-analysis and was significant at 389 the suggestive level $\left(\mathrm{p}<5 \times 10^{-5}\right)$ in both genders.

390 This declaration of a replicated association was approximately same as a two-stage design,

391 where the first stage involves selecting variants at the suggestive level $\left(\mathrm{p}<5 \times 10^{-5}\right)$ in one gender

392 and the second stage involves replicating the selected variants at the same significance level

$393\left(\mathrm{p}<5 \times 10^{-5}\right)$ in another gender. An association locus was defined as a genomic region of $500 \mathrm{~kb}$ to

394 both sides of a significant lead signal.

395 Difference in effect size between female and male was examined by a two-tailed p-value from

396 the z-score in the following equation

$$
z=\frac{\beta_{\text {female }}-\beta_{\text {male }}}{\sqrt{\operatorname{var}\left(\beta_{\text {female }}\right)+\operatorname{var}\left(\beta_{\text {male }}\right)}}
$$

397 , where $\beta_{\text {female }}$ and $\beta_{\text {male }}$ are regression coefficients for females and males, and $\operatorname{var}(\cdot)$ are their

398 variances, respectively.

\section{Conditional association analysis}

400 To identify additional signals in regions of association, approximate joint and conditional 401 association analysis was performed in each region using the GCTA tool [55].

403 From the UKB sample, a reference sample of 100,000 unrelated subjects was generated for 404 estimating LD pattern for subsequent analyses. The unrelated subjects were inferred with KING 405 software [56], from whom the 100,000 subjects of the reference sample were randomly drawn. 406 Quality control (QC) procedures applied to the reference sample included Hardy-Weinberg 407 equilibrium $\left(\mathrm{p}>1 \times 10^{-6}\right)$ and $\mathrm{MAF}>0.1 \%$. 
A recursive conditional association analysis was performed. In each iteration, an approximate

409 conditional analysis conditioning on the current list of lead variants was performed in each

410 gender, followed by an across-gender meta-analysis to combine the gender-specific results.

411 Again, a significant replicated association was defined as achieving both a conditional

412 meta-analysis GWS signal $\left(\mathrm{p}<5 \times 10^{-9}\right)$ and a conditional suggestive signal $\left(\mathrm{p}<5 \times 10^{-5}\right)$ in both

413 genders. In addition, each such identified variant is required to be independent of all variants in

414 the lead SNP list (LD $\left.\mathrm{r}^{2}<0.1\right)$. The variant with the lowest $\mathrm{p}$-value among such identified ones

415 was added into the list of lead variants. Iterations of the conditional analysis were run until no

416 significant signal can be identified.

\section{Overlap with loci in previous GWAS of obesity traits}

418 GWAS summary statistics for 5 obesity traits, including body mass index (BMI) [57], waist

419 circumference (WC), WC adjusted for BMI ( $\left.\mathrm{WC}_{\text {adj }} \mathrm{BMI}\right)$, waist-hip ratio (WHR) and WHR

420 adjusted for BMI (WHR ${ }_{\text {adj }} \mathrm{BMI}$ ) [58], were downloaded from the GIANT consortium website.

421 For each trait, SNPs located within all the 561 identified loci (lead SNP +500 kb flanking region

422 at each side) were extracted from the GWAS summary statistics. Significance level for the

423 obesity traits were set at the conventional level of $5.0 \times 10^{-8}$.

\section{Exome sequencing association analysis}

425 During the preparation of this manuscript, the UKB released exome-sequencing data on a 426 selected subset of $\sim 50,000$ participants. We compared the exome-sequencing based association

427 results with that based on genotype imputation. To accomplish this, we generated an unrelated

428 sample consisting of subjects who were both exome-sequenced and genotype-imputed.

429 As the QC procedure, we removed subjects who were not self-reported as white, whose 430 self-reported genders were inconsistent with their genetic genders, and who withdrew their 
431 consents. The KING software was used to select unrelated subjects based on pairwise kinship

432 matrix for up to $2^{\text {nd }}$ degree relatedness [56]. The final sample consisted of 45,554 participants,

433 including 24,740 females and 20,814 males.

434 Sequence variant coordinates, which were annotated to the GRCH38 assembly, were 435 converted back to the GRCH37 assembly with Liftover

436 (http://genome.ucsc.edu/cgi-bin/hgLiftOver). For each subject, variants that were missing in the 437 sequenced data were set to missing in the imputed data as well. In both datasets, genetic 438 association with normalized phenotype residuals was analyzed with PLINK2 [59]. The top 10

439 PCs were included as covariates to account for potential population stratification.

\section{Genetic architecture}

441 BOLT-REML was used to estimate heritability tagged by all the analyzed variants [18]. LD

442 score regression (LDSC) method was used to estimate the amount of genomic inflation due to

443 confounding factors such as population stratification and cryptic relatedness [17]. Pre-computed

444 LD scores from the 1000 Genomes project European subjects were used for estimation. The

445 relative contribution of confounding factors was measured by attenuation ratio (AR), which is

446 defined as (intercept-1)/(mean $\left.\operatorname{chi}^{\wedge} 2-1\right)$, where intercept and mean $\operatorname{chi}^{\wedge} 2$ are estimates of

447 confounding and the overall association inflation, respectively [17].

448 To compare AR with that estimated on unrelated subjects, a maximal subset of unrelated 449 subjects from the total sample being analyzed were generated. Specifically, KING was used to 450 extract a subset of unrelated subjects [56]. The resulting unrelated sample included 369,968 451 participants (198,989 females and 170,979 males). In each gender, PLINK2 was used to perform 452 genetic association analysis [59]. To account for genetic confounding, the top 10 PCs inferred 453 from UKB were used as the additional covariates. 
454 To calculate the variance explained by all independent lead variants, individual variant effect

455 size was estimated with the formula $2 f(1-f) \beta^{2}$, where $f$ is allele frequency and $\beta$ is regression

456 coefficient associated with the variant.

\section{$457 \quad$ Enrichment analysis}

458 Stratified LDSC was used to partition heritability from GWAS summary statistics into

459 different functional categories [21]. The analysis was based on the 'full baseline model' created

460 by Finucane et al. [21] from 24 publicly available main annotations that are not specific to any

461 cell type. Significance level of enrichment was set at $\mathrm{p}<2.08 \times 10^{-3}(0.05 / 24)$.

462 The stratified LDSC was used to also assess the enrichment of heritability into specific tissues

463 and cell types [22]. This method analyzes gene expression data together with GWAS summary

464 statistics, for which, the two pre-compiled gene expression datasets in LDSC were used. The first

465 one is the GTEx project v6p [23] and the second one is the Franke lab dataset [24]. The GTEx

466 dataset contains 53 tissues with an average of 161 samples per tissue. The Franke lab dataset is

467 an aggregation of publicly available microarray gene expression datasets comprising 37,427

468 human samples from 152 tissues. The total $205(=53+152)$ tissues are classified into nine

469 categories for visualization. Significance was declared at a false discovery rate (FDR) $<5 \%$.

\section{Candidate gene prioritization}

471 In each associated locus, a set of credible risk variants (CRVs) were defined as those variants

472 in strong LD with the lead variant $\left(r^{2}>0.8\right.$, including lead variant $)$. LD $r^{2}$ measure was estimated

473 based on the 100,000 unrelated reference sample with LDstore [60]. Six sources of information

474 was used to evaluate a gene's causality: 1) being nearest to the lead CRV; 2) containing a

475 mis-sense coding CRV; 3) being a target gene for a cis-eQTL CRV; 4) being a target gene for a 
476 cis- protein QTL (cis-pQTL) CRV; 5) being prioritized by DEPICT analysis [27] and 6) being

477 prioritized by SMR analysis [61].

478 Cis-eQTLs revealed by the GTEx (v7) project were accessed from the GTEx web portal

479 (www.gtexportal.org/) [23]. Cis-eQTL information is available for over 50 tissues. We selected

480 skeletal muscle and whole blood for our analysis. Cis-eQTL was searched within $500 \mathrm{~kb}$ distance

481 from a target gene. Significant cis-eQTL was declared at $\mathrm{p}<5 \times 10^{-5}$.

482 Cis-pQTL information was accessed from Sun et al. [25]. GWAS summary statistics for 3,284

483 proteins were downloaded from the study's website. Cis-pQTL was searched within $500 \mathrm{~kb}$

484 distance from a target gene. Significant cis-eQTL was declared at $\mathrm{p}<5 \times 10^{-5}$.

485 DEPICT is an integrative tool that takes advantage of predicted gene functions to

486 systematically prioritize the most likely causal genes at loci of interest [27]. The input of

487 DEPICT includes a list of variant identifiers, and the output contains all genes located in the loci

488 and their p-values of being a candidate gene. All lead variants were submitted to DEPICT for 489 analysis. Significant genes were declared at a false discovery rate (FDR) $<5 \%$.

490 SMR (Summary data-based Mendelian Randomization) method [61] is another SNP 491 prioritization program that integrates summary-level data from GWAS with data from eQTL 492 studies to identify genes whose expression levels are associated with trait due to causal or 493 pleiotropy effects. Here, the pleiotropy effect means that a SNP is causally associated with both 494 gene expression and phenotypic variation. SMR uses SNPs as an instrumental variable and tests 495 the causal relation of gene expression to phenotype variation. The results are interpreted as the 496 effect of gene expression on phenotype free of confounding from non-genetic factors. We used a 497 pre-compiled eQTL dataset in whole blood tissue [62] for estimation.

\section{Gene-based and gene set enrichment analyses}


Gene-based association analysis was performed with MAGMA v1.6 [63], as implemented on

500 the FUMA website (http://fuma.ctglab.nl/). GWAS meta-analysis summary statistics were

501 mapped to 19,427 protein-coding genes, resulting in 19,098 genes that were covered by at least

502 one SNP. Gene-based association test was performed taking into account the LD between

503 variants. Gene-based significance level was set at stringent Bonferroni corrected threshold

$5042.62 \times 10^{-6}$, i.e., $0.05 / 19,098$.

505 The generated gene-based summary statistics were further used to test for enrichment of

506 association to specific biological pathways or gene sets. A gene set's association signal was

507 evaluated by integrating all signals from the genes in the set with MAGMA [63]. A competitive

508 gene set analysis model was used to test whether the genes in a gene set are more strongly

509 associated with the phenotype than other genes.

510 Gene sets were obtained through the MSigDB website

511 (http://software.broadinstitute.org/gsea/msigdb/index.jsp) [64]. Each gene was assigned to a gene

512 set as annotated by gene ontology (GO), Kyoto encyclopedia of genes and genomes (KEGG),

513 Reactome and BioCarta gene set databases and other gene sets curated by domain experts or

514 biomedical literature [64]. A total of 10,651 (4,734 curated and 5,917 GO terms) gene sets were

515 used in this analysis. The significance level was set at a Bonferroni-corrected level of $5160.05 / 10,651=4.69 \times 10^{-6}$

517 Protein-protein interaction network was constructed with STRING [65]. STRING uses

518 information based on gene co-expression, text-mining, and others, to construct protein interactive

519 network.

$520 \quad$ Polygenic risk score profiling 
To assess the capability of the GWAS finding to predict ALM, a polygenic scoring analysis

522 was conducted in the 369,968 unrelated subjects extracted from the main UKB sample. Three

523 quarters of the individuals (277,762 subjects, including 149,329 females) were randomly selected

524 as the training sample, and the remaining one quarter individuals $(92,206$ participants, including

52549,660 females) as the validation sample. Female and male subjects were pooled together for

526 analysis.

527 Raw phenotype was adjusted by age, age ${ }^{2}$, gender, height, height ${ }^{2}$ and the top 10 PCs, and the

528 residuals were converted to the standard normal distribution quantiles for downstream analysis.

529 Genetic association analysis was performed with PLINK2 [59].

530 The same QC procedures as in the main analysis were used to process the variants. The

531 variants achieving a p-value of $<1 \times 10^{-5}$ in the training sample were selected and used for

532 prediction in the validation sample via LDpred approach [66]. LDpred infers the posterior mean

533 effect size of each marker by using a prior on effect sizes and LD information from an external

534 reference panel. Specifically, the validation sample with original genotypes was used as

535 reference panel for LD estimation. The number of SNPs used to adjust LD from each side of the

536 target SNP was set to 1000. Other software parameters were set to the default.

\section{Genetic correlations with other traits}

538 To test whether lean mass has a shared genetic etiology with other diseases and relevant traits,

539 a genetic correlation analysis was performed with LDSC method [17]. An online web tool,

540 LDHub (http://ldsc.broadinstitute.org/ldhub/), was used to estimate the genetic correlation

541 between $\mathrm{ALM}_{\text {adj }}$ and 49 complex traits and diseases. The standalone version of the software was

542 used to estimate between $\mathrm{ALM}_{\mathrm{adj}}$ and two additional traits, ALM and total body lean mass,

543 measured by the DXA scan, which are not available in the LDHub GWAS summary statistics 
544 collections, and which were downloaded from the GEFOS consortium website

545 (http://www.gefos.org).

546 Both the LDHub and standalone analyses adopted same QC criteria. Specifically, only

547 HapMap3 autosomal SNPs were included to minimize poor imputation quality [17]. SNPs were

548 further removed given the following conditions: $\mathrm{MAF}<0.01$, ambiguous strand $(\mathrm{A} / \mathrm{T}$ or $\mathrm{C} / \mathrm{G})$,

549 duplicated identifier, or reported sample size less than $60 \%$ of total sample size. LD scores

550 pre-computed on the 1000 genomes project European individuals were used for calculation.

\section{Mendelian randomization analysis}

552 To investigate whether ALM (as exposure) is causally associated with complex diseases (as

553 outcome), a Mendelian randomization analysis with GSMR was performed [26] on selected 10

554 complex diseases, including fracture [36], type 2 diabetes (T2D) [37], asthma [38], insomnia

555 [39], inflammatory bowel disease (IBD) [40], smoking addiction [41], coronary artery disease

556 (CAD) [42], amyotrophic lateral sclerosis (ALS) [43], bipolar disorder [44] and autistic spectrum

557 disorder (ASD) [45].

558 GWAS summary statistics for these diseases were downloaded from the respective websites.

559 From the list of SNPs whose association signals with $\mathrm{ALM}_{\mathrm{adj}}$ were below $5 \times 10^{-8}$, qualified SNPs

560 were included based on the following criteria: concordant alleles between exposure and outcome

561 GWAS summary statistics, non-palindromic SNPs with certain strand, MAF>1\%, and allele

562 frequency difference between exposure and outcome GWAS summary statistics $<0.2$.

563 Independent SNPs were further clumped with PLINK2 [59] with independence LD threshold

$564 \mathrm{r}^{2}<0.05$ and $1 \mathrm{MB}$ window size. The clumped independent SNPs were examined for their

565 pleiotropic effects to both exposure and outcome by the HEIDI test [26]. Significance level for

566 the HEIDI test was set to $\alpha=1 \times 10^{-5}$. After removing pleiotropic SNPs, the remaining independent 
567 SNPs were taken as instrumental variables to test for the causal effect of exposure to outcome.

568 The estimated causal effect coefficients are approximately equal to the natural log odds ratio (OR)

569 for a case-control trait. The MR analysis significance level was set to $0.005(0.05 / 10)$. 
572 This research was conducted using the UK Biobank resource under application number 41542.

573 This study was partially supported by the funding from national natural science foundation of

574 China (31571291 to LZ, 31771417 and 31501026 to YFP), the natural science foundation of

575 Jiangsu province of China (BK20150323 to YFP).

576 
References

1. Giles, J.T., et al., Association of body composition with disability in rheumatoid arthritis: impact of appendicular fat and lean tissue mass. Arthritis Rheum, 2008. 59(10): p. 1407-15.

2. Janssen, I., S.B. Heymsfield, and R. Ross, Low relative skeletal muscle mass (sarcopenia) in older persons is associated with functional impairment and physical disability. J Am Geriatr Soc, 2002. 50(5): p. 889-96.

3. Miyakoshi, N., et al., Prevalence of sarcopenia in Japanese women with osteopenia and osteoporosis. J Bone Miner Metab, 2013. 31(5): p. 556-61.

4. Binkley, N., D. Krueger, and B. Buehring, What's in a name revisited: should osteoporosis and sarcopenia be considered components of "dysmobility syndrome?". Osteoporos Int, 2013. 24(12): p. 2955-9.

5. Wannamethee, S.G. and J.L. Atkins, Muscle loss and obesity: the health implications of sarcopenia and sarcopenic obesity. Proc Nutr Soc, 2015. 74(4): p. 405-12.

591 6. Evans, W.J., et al., Cachexia: a new definition. Clin Nutr, 2008. 27(6): p. 793-9.

7. Brown, J.C., M.O. Harhay, and M.N. Harhay, Sarcopenia and mortality among a population-based sample of community-dwelling older adults. J Cachexia Sarcopenia Muscle, 2016. 7(3): p. 290-8.

8. Arden, N.K. and T.D. Spector, Genetic influences on muscle strength, lean body mass, and bone mineral density: a twin study. J Bone Miner Res, 1997. 12(12): p. 2076-81.

9. Livshits, G., et al., Contribution of Heritability and Epigenetic Factors to Skeletal Muscle Mass Variation in United Kingdom Twins. J Clin Endocrinol Metab, 2016. 101(6): p. 2450-9.

10. Liu, X.G., et al., Genome-wide association and replication studies identified TRHR as an important gene for lean body mass. Am J Hum Genet, 2009. 84(3): p. 418-23.

11. Ran, S., et al., Gene-based genome-wide association study identified 19p13.3 for lean body mass. Sci Rep, 2017. 7: p. 45025.

12. Hai, R., et al., Bivariate genome-wide association study suggests that the DARC gene influences lean body mass and age at menarche. Sci China Life Sci, 2012. 55(6): p. 516-20.

13. Urano, T., et al., Large-scale analysis reveals a functional single-nucleotide polymorphism in the 5'-flanking region of PRDM16 gene associated with lean body mass. Aging Cell, 2014. 13(4): p. 739-43.

14. Klimentidis, Y.C., et al., Genetic Variant in ACVR2B Is Associated with Lean Mass. Med Sci Sports Exerc, 2016. 48(7): p. 1270-5.

15. Zillikens, M.C., et al., Large meta-analysis of genome-wide association studies identifies five loci for lean body mass. Nat Commun, 2017. 8(1): p. 80.

16. Loh, P.R., et al., Efficient Bayesian mixed-model analysis increases association power in large cohorts. Nat Genet, 2015. 47(3): p. 284-90.

17. Bulik-Sullivan, B.K., et al., $L D$ Score regression distinguishes confounding from polygenicity in genome-wide association studies. Nature Genetics, 2015. 47(3): p. 291-295.

18. Loh, P.R., et al., Contrasting genetic architectures of schizophrenia and other complex diseases using fast variance-components analysis. Nat Genet, 2015. 47(12): p. 1385-92. 
621 19. Medina-Gomez, C., et al., Bivariate genome-wide association meta-analysis of pediatric musculoskeletal traits reveals pleiotropic effects at the SREBF1/TOM1L2 locus. Nat Commun, 2017. 8(1): p. 121.

20. Hubel, C., et al., Genomics of body fat percentage may contribute to sex bias in anorexia nervosa. Am J Med Genet B Neuropsychiatr Genet, 2018.

21. Finucane, H.K., et al., Partitioning heritability by functional annotation using genome-wide association summary statistics. Nat Genet, 2015. 47(11): p. 1228-35.

22. Finucane, H.K., et al., Heritability enrichment of specifically expressed genes identifies disease-relevant tissues and cell types. Nat Genet, 2018. 50(4): p. 621-629.

23. Consortium, G.T., Human genomics. The Genotype-Tissue Expression (GTEx) pilot analysis: multitissue gene regulation in humans. Science, 2015. 348(6235): p. 648-60.

24. Fehrmann, R.S., et al., Gene expression analysis identifies global gene dosage sensitivity in cancer. Nat Genet, 2015. 47(2): p. 115-25.

25. Sun, B.B., et al., Genomic atlas of the human plasma proteome. Nature, 2018. 558(7708): p. 73-79.

26. Zhu, Z., et al., Causal associations between risk factors and common diseases inferred from GWAS summary data. Nat Commun, 2018. 9(1): p. 224.

27. Pers, T.H., et al., Biological interpretation of genome-wide association studies using predicted gene functions. Nat Commun, 2015. 6: p. 5890.

28. Adzhubei, I.A., et al., A method and server for predicting damaging missense mutations. Nat Methods, 2010. 7(4): p. 248-9.

29. Kumar, P., S. Henikoff, and P.C. Ng, Predicting the effects of coding non-synonymous variants on protein function using the SIFT algorithm. Nat Protoc, 2009. 4(7): p. 1073-81.

30. Choi, Y., et al., Predicting the functional effect of amino acid substitutions and indels. PLoS One, 2012. 7(10): p. e46688.

31. Shihab, H.A., et al., Predicting the functional, molecular, and phenotypic consequences of amino acid substitutions using hidden Markov models. Hum Mutat, 2013. 34(1): p. 57-65.

32. Schmidt, K., et al., Sox8 is a specific marker for muscle satellite cells and inhibits myogenesis. J Biol Chem, 2003. 278(32): p. 29769-75.

33. Ge, X., et al., Lack of Smad3 signaling leads to impaired skeletal muscle regeneration. Am J Physiol Endocrinol Metab, 2012. 303(1): p. E90-102.

34. Estrella, N.L., et al., MEF2 transcription factors regulate distinct gene programs in mammalian skeletal muscle differentiation. J Biol Chem, 2015. 290(2): p. 1256-68.

35. Frank, N.Y., et al., Regulation of myogenic progenitor proliferation in human fetal skeletal muscle by BMP4 and its antagonist Gremlin. J Cell Biol, 2006. 175(1): p. 99-110.

36. Trajanoska, K., et al., Assessment of the genetic and clinical determinants of fracture risk: genome wide association and mendelian randomisation study. BMJ, 2018. 362: p. k3225.

37. Xue, A., et al., Genome-wide association analyses identify 143 risk variants and putative regulatory mechanisms for type 2 diabetes. Nat Commun, 2018. 9(1): p. 2941.

38. Moffatt, M.F., et al., A large-scale, consortium-based genomewide association study of asthma. N Engl J Med, 2010. 363(13): p. 1211-1221. 
663

664

665

666

667

668

669

670

671

672

673

674

675

676

677

678

679

680

681

682

683

684

685

686

687

688

689

690

691

692

693

694

695

696

697

698

699

700

701

702

703

704

705

39. Hammerschlag, A.R., et al., Genome-wide association analysis of insomnia complaints identifies risk genes and genetic overlap with psychiatric and metabolic traits. Nat Genet, 2017. 49(11): p. 1584-1592.

40. Liu, J.Z., et al., Association analyses identify 38 susceptibility loci for inflammatory bowel disease and highlight shared genetic risk across populations. Nat Genet, 2015. 47(9): p. 979-986.

41. Genome-wide meta-analyses identify multiple loci associated with smoking behavior. Nat Genet, 2010. 42(5): p. 441-7.

42. van der Harst, P. and N. Verweij, Identification of 64 Novel Genetic Loci Provides an Expanded View on the Genetic Architecture of Coronary Artery Disease. Circ Res, 2018. 122(3): p. 433-443.

43. van Rheenen, W., et al., Genome-wide association analyses identify new risk variants and the genetic architecture of amyotrophic lateral sclerosis. Nat Genet, 2016. 48(9): p. 1043-8.

44. Stahl, E.A., et al., Genome-wide association study identifies 30 Loci Associated with Bipolar Disorder. bioRxiv, 2018: p. 173062.

45. Grove, J., et al., Identification of common genetic risk variants for autism spectrum disorder. Nat Genet, 2019. 51(3): p. 431-444.

46. Manolio, T.A., et al., Finding the missing heritability of complex diseases. Nature, 2009. 461(7265): p. 747-53.

47. Zuk, O., et al., The mystery of missing heritability: Genetic interactions create phantom heritability. Proc Natl Acad Sci U S A, 2012. 109(4): p. 1193-8.

48. Yang, J., et al., Common SNPs explain a large proportion of the heritability for human height. Nat Genet, 2010. 42(7): p. 565-9.

49. Wood, A.R., et al., Defining the role of common variation in the genomic and biological architecture of adult human height. Nat Genet, 2014. 46(11): p. 1173-86.

50. Consortium, S.W.G.o.t.P.G., Biological insights from 108 schizophrenia-associated genetic loci. Nature, 2014. 511(7510): p. 421-7.

51. Karasik, D. and D.P. Kiel, Genetics of the musculoskeletal system: a pleiotropic approach. J Bone Miner Res, 2008. 23(6): p. 788-802.

52. Blank, R.D., Bone and Muscle Pleiotropy: The Genetics of Associated Traits. Clin Rev Bone Miner Metab, 2014. 12(2): p. 61-65.

53. Loh, P.R., et al., Mixed-model association for biobank-scale datasets. Nat Genet, 2018. 50(7): p. 906-908.

54. Sanna, S., et al., Common variants in the GDF5-UQCC region are associated with variation in human height. Nat Genet, 2008. 40(2): p. 198-203.

55. Yang, J., et al., GCTA: a tool for genome-wide complex trait analysis. Am J Hum Genet, 2011. 88(1): p. 76-82.

56. Manichaikul, A., et al., Robust relationship inference in genome-wide association studies. Bioinformatics, 2010. 26(22): p. 2867-73.

57. Yengo, L., et al., Meta-analysis of genome-wide association studies for height and body mass index in approximately 700000 individuals of European ancestry. Hum Mol Genet, 2018. 27(20): p. 3641-3649. 
58. Shungin, D., et al., New genetic loci link adipose and insulin biology to body fat distribution. Nature, 2015. 518(7538): p. 187-196.

708 59. Chang, C.C., et al., Second-generation PLINK: rising to the challenge of larger and richer datasets. Gigascience, 2015. 4: p. 7.

60. Benner, C., et al., Prospects of Fine-Mapping Trait-Associated Genomic Regions by Using Summary Statistics from Genome-wide Association Studies. Am J Hum Genet, 2017. 101(4): p. 539-551.

61. Zhu, Z., et al., Integration of summary data from GWAS and eQTL studies predicts complex trait gene targets. Nat Genet, 2016. 48(5): p. 481-7.

62. Westra, H.J., et al., Systematic identification of trans eQTLs as putative drivers of known disease associations. Nat Genet, 2013. 45(10): p. 1238-1243.

63. de Leeuw, C.A., et al., MAGMA: generalized gene-set analysis of GWAS data. PLoS Comput Biol, 2015. 11(4): p. e1004219.

64. Subramanian, A., et al., Gene set enrichment analysis: a knowledge-based approach for interpreting genome-wide expression profiles. Proc Natl Acad Sci U S A, 2005. 102(43): p. 15545-50.

65. Szklarczyk, D., et al., STRING v11: protein-protein association networks with increased coverage, supporting functional discovery in genome-wide experimental datasets. Nucleic Acids Res, 2019. 47(D1): p. D607-D613.

66. Vilhjalmsson, B.J., et al., Modeling Linkage Disequilibrium Increases Accuracy of Polygenic Risk Scores. Am J Hum Genet, 2015. 97(4): p. 576-92. 


\section{Figure Legends}

\section{Figure 1. Main association results.}

732 Figure 1A, Per allele effect size versus minor allele frequency (MAF). X-axis is MAF at the 717

733 identified variants and y-axis is per allele effect size (regression coefficient). Figure 1B, the Manhattan

734 plot of the meta-analysis combining both genders. The horizontal red line indicates the genome-wide

735 significance level (alpha $\left.=5 \times 10^{-9}\right)$ in $-\log _{10}$ scale. All novel loci were marked in green.

737 Figure 2. Heritability enrichment in different functional annotations and tissues.

738 Figure $\mathbf{2 A}$ is enrichment of genome-wide association signals in 24 main annotations using LDSC

739 regression. Y-axis represents the ratio of phenotypic variance explained by variants in a particular 740 annotation category against that explained in the remaining regions. Error bars represent jackknife

741 standard errors around the estimates of enrichment. A single asterisk indicates significance at $\mathrm{p}<0.05$ after

742 Bonferroni correction for the 24 hypotheses tested, and two asterisks indicates significance at $\mathrm{p}<0.01$.

743 Figure 2B is enrichment of genome-wide association signals in 206 cells/tissues from two different

744 databases (Franke lab dataset and GTEx consortium dataset). The total cells/tissues were divided into

7459 categories. Each dot represents a specific cell/tissue and the tissues passing the cutoff of FDR $<5 \%$ at

$746-\log 10(\mathrm{p})=2.75$ were marked in large.

748 Figure 3. Protein-protein interactional network.

749 Thirty-four genes over-represented in 85 significant pathways were selected to construct a protein-protein

750 interaction network with STRING, which bases the construction on knowledge of gene co-expression, 751 text-mining, and others.

753 Figure 4. Polygenic score prediction. 
754 A total of 277,762 subjects were randomly selected as the training sample, and another

755 independent 92,206 subjects were selected as the validation sample. The variants achieving a

$756 \mathrm{p}$-value of $<1 \times 10^{-5}$ in the training sample were selected and used for prediction in the validation

757 sample via LDpred approach. Subjects in the two extreme tails of the predicted genome-wide

758 polygenic score (GPS) distribution were compared in terms of raw phenotype mean (after

759 correction). X-axis represents the fraction of subjects drawn from both extreme tails of the predicted

760 GPS distribution. Y-axis represents mean $\operatorname{ALM}_{\text {adj }}( \pm 95 \%$ confidence interval).

761

762 Figure 5. Genetic overlap with other traits.

763 Genetic correlations $\left(\mathrm{r}_{\mathrm{g}}\right)$ between $\mathrm{ALM}_{\text {adj }}$ and 51 traits and diseases were estimated. LD Score regression

764 tested genome-wide SNP associations for these participants against similar data for various other traits

765 and diseases containing Musculoskeletal system, anthropometrics, obesity, cognition, metabolism,

766 psychiatry, reproduction and neuropsychiatric outcomes. Error bars represent standard errors on these

767 estimates. Blue bars represent significantly positive correlation at the nominal level $\mathrm{p}<0.05$; pink bars

768 represent significantly negative correlation $(\mathrm{p}<0.05)$; grey bars represent non-significant correlation. 
Table 1. Association results of 42 mis-sense variants.

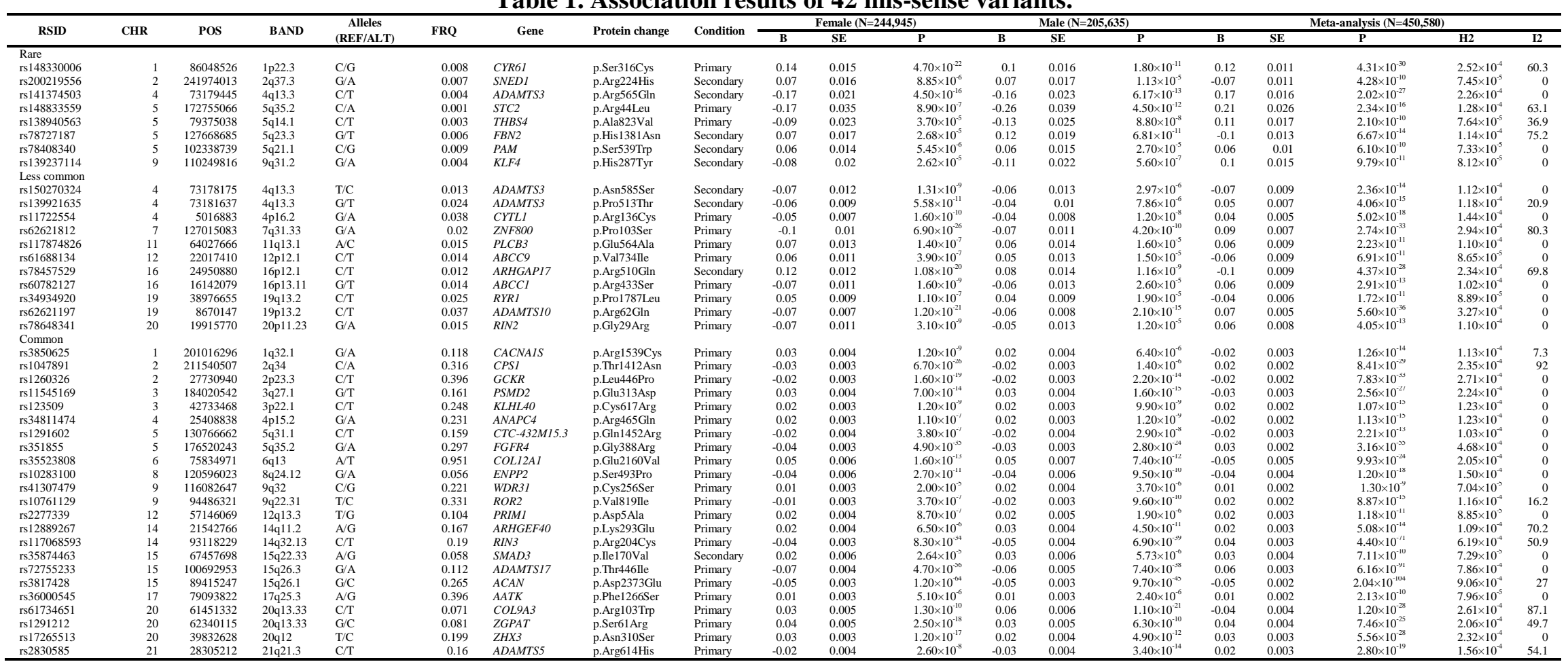

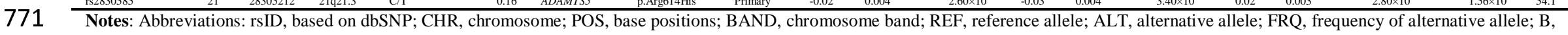

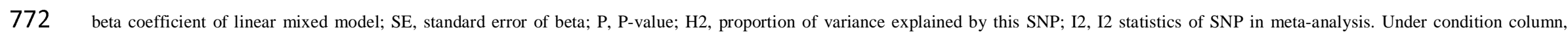

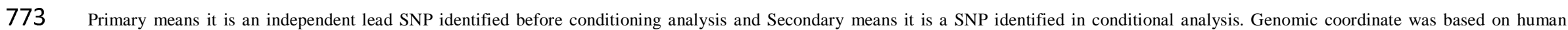

774 genome assembly build 37 (GRCh 37). 
A
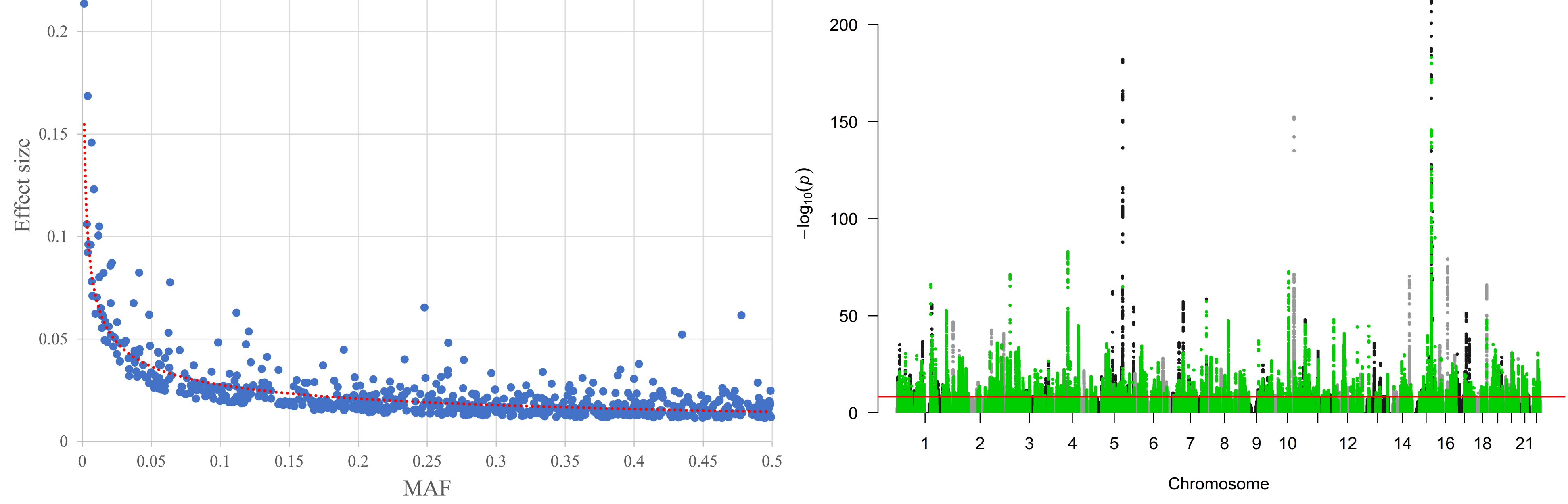

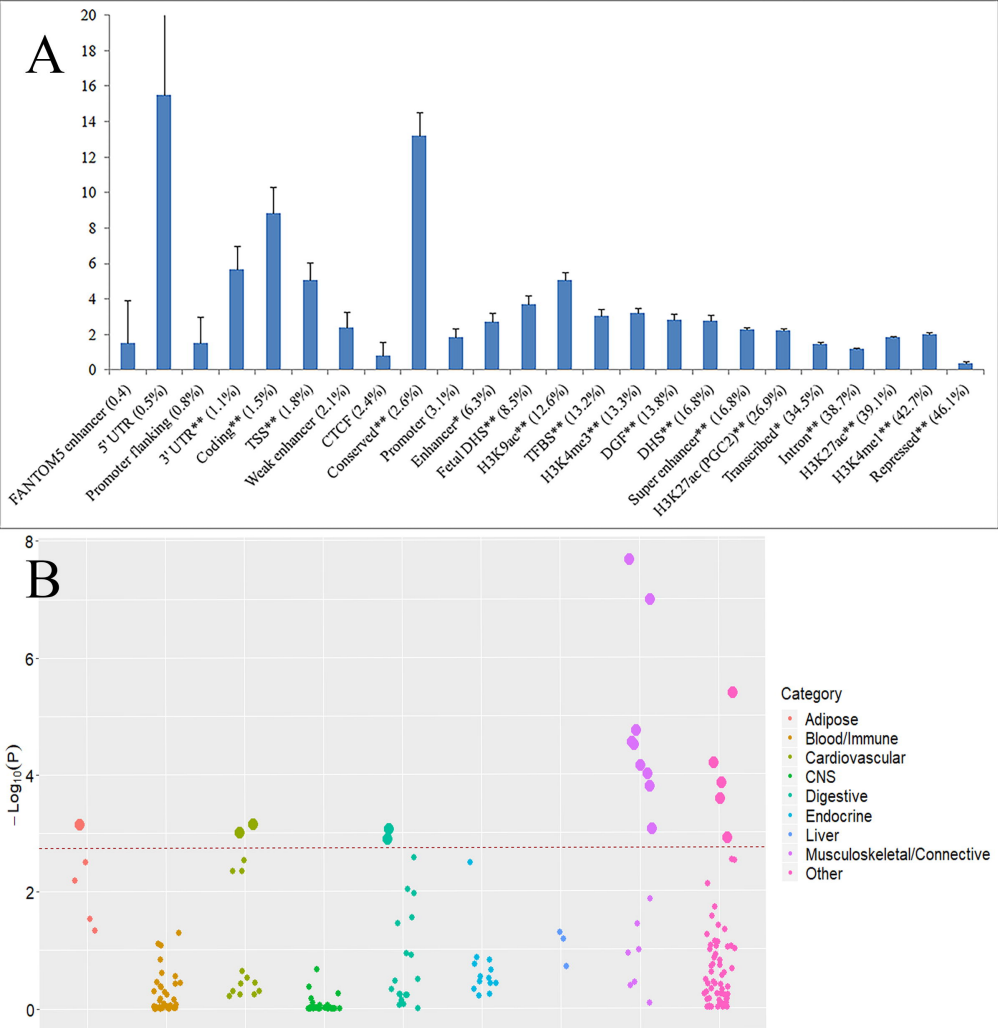

Category

- Adipose

Blood/Immune

- Cardiovascular

- CNS

- Digestive

- Endocrine

- Liver

- Musculoskeletal/Connective

- Other 


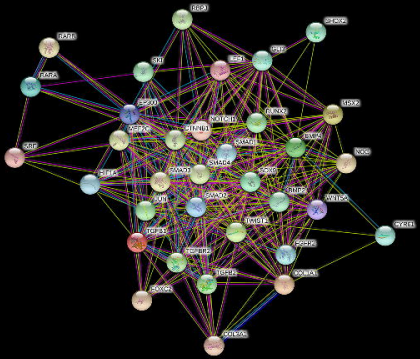




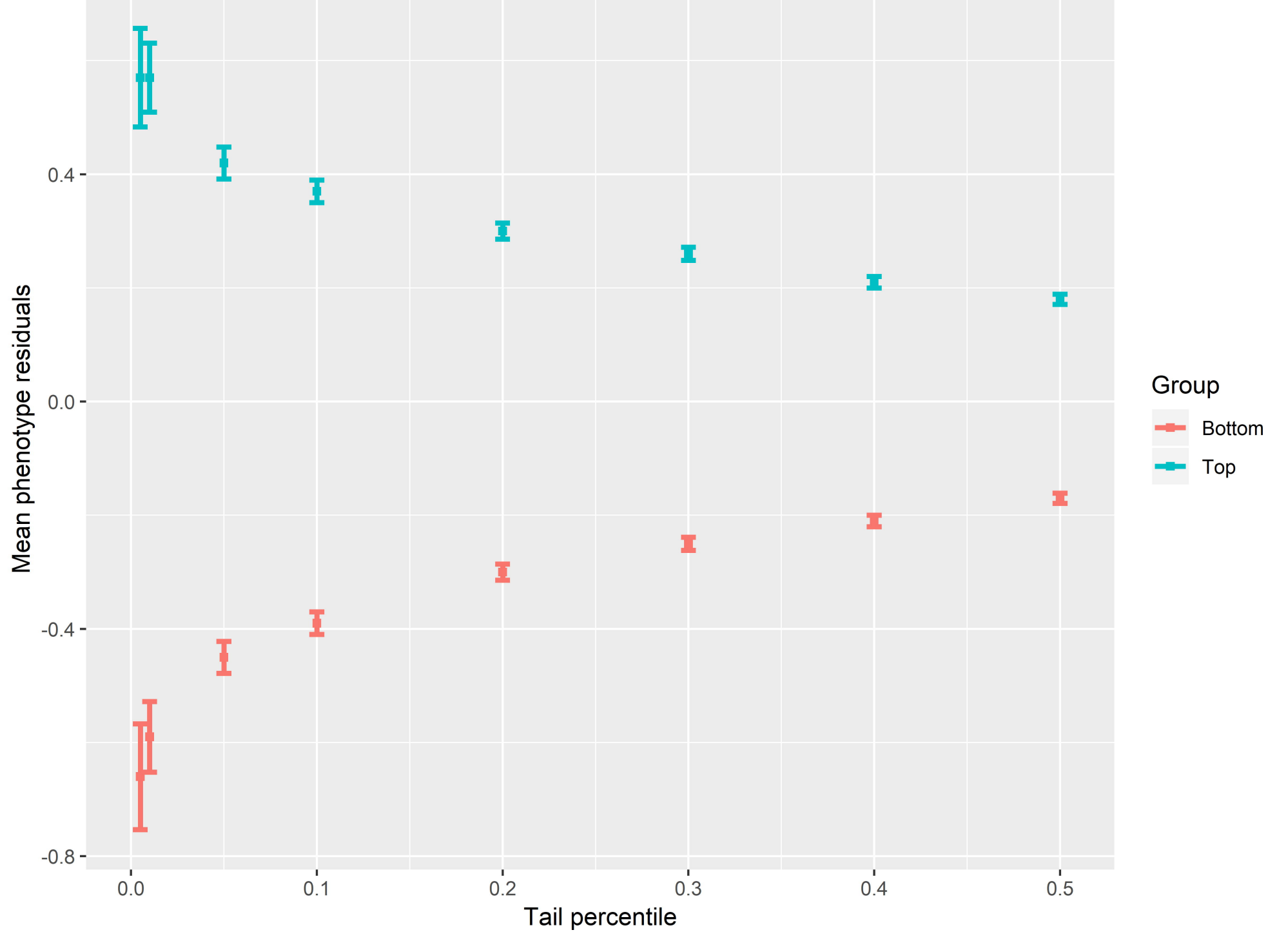




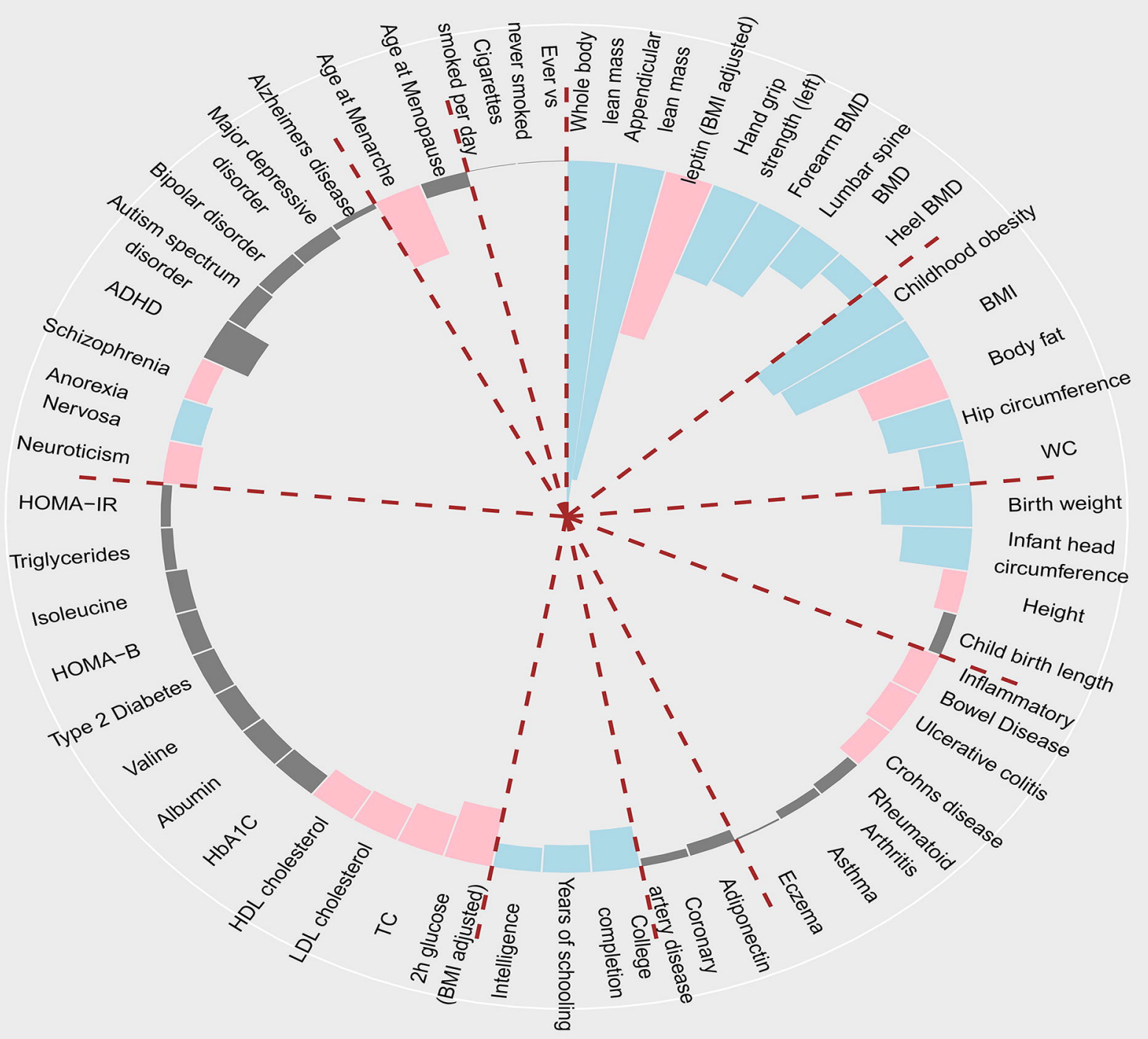

Direction

Not significant Positive

Negative 